\title{
Evaluating genetic diversity and identifying priority conservation for seven Tibetan pig populations in China based on the mtDNA D-loop
}

\author{
Qianyun Ge1, Caixia Gao², Yuan Cai', Ting Jiao ${ }^{3}$, Jinqiang Quan', \\ Yongbo Guo ${ }^{4}$, Wangshan Zheng ${ }^{4}$, and Shengguo Zhao ${ }^{1, *}$
}

\footnotetext{
* Corresponding Author: Shengguo Zhao Tel: +86-139-09469015, Fax: +86-931-7632468, E-mail: zhaoshengguo0628@hotmail.com

${ }^{1}$ College of Animal Science \& Technology, Gansu Agricultural University, Lanzhou 730070, China

2 State Key Laboratory of Veterinary Biotechnology, Harbin Veterinary Research Institute, Chinese Academy of Agricultural Sciences, Harbin 150069, China

${ }^{3}$ College of Grassland, Gansu Agricultural University, Lanzhou 730070, China

${ }^{4}$ State Key Laboratory of Genetic Resources and Evolution, Kunming Institute of Zoology, Chinese Academy of Sciences, Kunming 650223, China
}

ORCID

Qianyun Ge

https://orcid.org/0000-0002-1255-6829

Caixia Gao

https://orcid.org/0000-0001-8183-9753

Yuan Cai

https://orcid.org/0000-0003-2828-2794

Ting Jiao

https://orcid.org/0000-0003-0839-5177 Jingiang Quan

https://orcid.org/0000-0001-5666-5915 Yongbo Guo

https://orcid.org/0000-0003-4588-1713

Wangshan Zheng

https://orcid.org/0000-0002-6174-4746

Shengguo Zhao

https://orcid.org/0000-0001-8744-375X

Submitted Sept 27, 2019; Revised Dec 13, 2019; Accepted Dec 21, 2019
Objective: Tibetan pigs, an excellent species unique to China, face serious threats, which in turn affects the development and utilization of the outstanding advantages of plateau hypoxia adaptability and reduces their genetic diversity. Therefore, a discussion of measures to conserve this genetic resource is necessary. The method, based on genetic diversity, genetic divergence and total genetic contribution rate of population, reflects the priority conservation order and varies depending on the three different purposes of conservation.

Methods: We analyzed mitochondrial DNA control region (D-loop) variation in 1,201 individuals from nine Tibetan pig populations across five provinces and downloaded 564 mtDNA D-loop sequences from three indigenous pig breeds in Qinghai, Sichuan, and Yunnan Provinces distributed near the Tibetan pigs.

Results: We analyzed three different aspects: Changdu Tibetan pigs have the highest genetic diversity, and from the perspective of genetic diversity, the priority conservation is Changdu Tibetan pigs. Hezuo Tibetan pigs have the highest genetic contribution, so the priority conservation is Hezuo Tibetan pigs in the genetic contribution aspect. Rkaze Tibetan pigs were severely affected by indigenous pig breeds, so if considering from the perspective of introgression, the priority conservation is Rkaze Tibetan pigs.

Conclusion: This study evaluated genetic diversity and comprehensively assessed conservation priority from three different aspects in nine Tibetan pig populations.

Keywords: Tibetan Pigs; mtDNA D-loop; Genetic Diversity; Conservation

\section{INTRODUCTION}

Tibetan pigs, an excellent species unique to China, are mainly distributed in the Tibetan Plateau area, which is the largest continuous high altitude ecosystem in the world, with an average altitude of more than $4,000 \mathrm{~m}$ [1]. These animals have a high status of genetic diversity in domestic pigs, but they also face serious threats, including loss habitat, because of increasing human population and activity, genetic introgression by crossbreeding with exotic breeds and intensifying production systems, which in turn affect the development and utilization of the outstanding advantages of plateau hypoxia adaptability and reduced genetic diversity. Although heterosis by crossing may improve economically important traits, undue crossbreeding may introduce DNA from other breeds and endanger the purity of some traditional local breeds [2], so genetic characterization of Tibetan pigs for conservation and rational use is therefore necessary and urgent. Due to differences in the size of populations, scarcity of funds for species conservation and conflict between conservation and economic development, deciding what and where to first conserve is an essential 
step in managing important species. Many researchers have studied issues, including genetic diversities in different populations [3], domestication centers [4], and migration history $[5,6]$, that have helped us understand the local and worldwide domestication history by using mitochondrial DNA (mtDNA). Vertebrate mtDNA is capable of self-replication and is strictly maternally inherited without recombination during generational transmission. Although mtDNA contains many functional genes and the genome length and structure are very conservative, but the primary structure changes are very active. mtDNA contains the displacement (D)-loop, which contains regulatory sequences controlling both replication and transcription of $\mathrm{mtDNA}$, is the fastestevolving region in mtDNA. Due to the high variation of mtDNA sequences, it can be effectively used to establish evolutionary relationships within species and between different populations. In this study, therefore, the genetic diversity of Tibetan pigs in nine populations across five provinces was comprehensively analyzed and assessed using the mtDNA D-loop, and priorities for conservation were discussed. The results of the study will contribute to the conservation and sustainable use of resources.

\section{MATERIALS AND METHODS}

\section{Sampling and sequencing}

Blood samples were collected from a total of 728 Tibetan pigs in seven different populations of Tibetan pigs where the samples had not been adequately collected before: Ganzi (79), Diqing (83), Linzhi (122), Shannan (72), Changdu (73), Hezuo (193), and Qinghai (106). All animal work was conducted according to the Institutional Animal Care and Use Committee (IACUC) and was approved by the Animal Care Committee of Gansu Agricultural University. Considering the limited quantity of testing sequences, an additional dataset of 473 samples was used to complement the data on representative Tibetan pigs to obtain high coverage of Tibetan pigs.
A total of $564 \mathrm{mtDNA}$ D-loop sequences from three indigenous pig breeds in Qinghai, Sichuan and Yunnan Provinces, which are located near where Tibetan pigs are distributed, were downloaded from GenBank and used as a reference set in this study. All sample summaries are listed in Table 1 (a list of collected and supplementary animals, with GenBank accession number and other detailed information, is provided in an editable format in Supplementary Table S1).

The D-loop region was amplified directly from the genomic DNA by polymerase chain reaction (PCR) using the primers 5'-CCAAAAACAAAGCAGAGTGTAC-3' and 5'-CGTTA TGAGCTACCGTTATA-3'. PCR was carried out in $25 \mu \mathrm{L}$ volumes and contained $12.5 \mu \mathrm{L}$ of $2 \times$ Eco Taq PCR Supermix containing $1 \mathrm{U}$ Taq polymerase, $500 \mathrm{mM}$ dNTPs, and 10× Taq buffer (Beijing TransGen Biotech Co., Ltd., Beijing, China), $0.1 \mu \mathrm{g}$ of template DNA, $0.4 \mu \mathrm{L}$ of each primer at 10 $\mathrm{pmol} / \mathrm{mL}$ and $11.6 \mu \mathrm{L}$ of $\mathrm{ddH}_{2} \mathrm{O}$. The cycling conditions were initial denaturation at $94^{\circ} \mathrm{C}$ for $5 \mathrm{~min}$, followed by 33 cycles of $94^{\circ} \mathrm{C}$ for $30 \mathrm{~s}, 56^{\circ} \mathrm{C}$ for $30 \mathrm{~s}$ and $72^{\circ} \mathrm{C}$ for $30 \mathrm{~s}$ and a final extension for $5 \mathrm{~min}$ at $72^{\circ} \mathrm{C}$ [7]. Amplified DNA fragments were purified following agarose gel electrophoresis and sequenced using the ABI 3130 DNA sequencer (Applied Biosystems, Foster City, CA, USA) [7].

\section{Data analysis}

Original sequence data were obtained using the ABI PRISM DNA sequencer software. Sequences were edited and aligned using ClustalX 1.81 [8]. MEGA 7.0 was used to collect sequences [9]. DnaSP 5.0 software was used to analyze the haplotypes and genetic diversity [7]. Correlation analysis and principal component analysis (PCA) were investigated by SPSS 19.0 .

\section{RESULTS}

\section{Genetic diversity analysis}

The 431-bp D-loop region of mtDNA was obtained. Haplo-

Table 1. Characteristics of samples

\begin{tabular}{|c|c|c|c|c|}
\hline Breed/Population & Abbreviation & Category & Sample size & Sampling site \\
\hline Aba Tibetan pig & AT & Tibetan & 70 & Aba, Sichuan \\
\hline Ganzi Tibetan pig & GT & Tibetan & 133 & Ganzi, Sichuan \\
\hline Diqing Tibetan pig & DT & Tibetan & 178 & Diqing, Yunan \\
\hline Linzhi Tibetan pig & $\mathrm{LT}$ & Tibetan & 241 & Linzhi, Tibet \\
\hline Shannan Tibetan pig & ST & Tibetan & 91 & Shannan, Tibet \\
\hline Changdu Tibetan pig & $\mathrm{CT}$ & Tibetan & 90 & Changdu, Tibet \\
\hline Rkaze Tibetan pig & RT & Tibetan & 24 & Rkaze, Tibet \\
\hline Hezuo Tibetan pig & HT & Tibetan & 268 & Hezuo, Gansu \\
\hline Qinghai Tibetan pig & QT & Tibetan & 106 & Qinghai \\
\hline Qinghai indigenous pig & $\mathrm{QH}$ & Indigenous & 115 & Qinghai \\
\hline Yunnan indigenous pig & YN & Indigenous & 219 & Yunnan \\
\hline Sichuan indigenous pig & SC & Indigenous & 230 & Sichuan \\
\hline
\end{tabular}




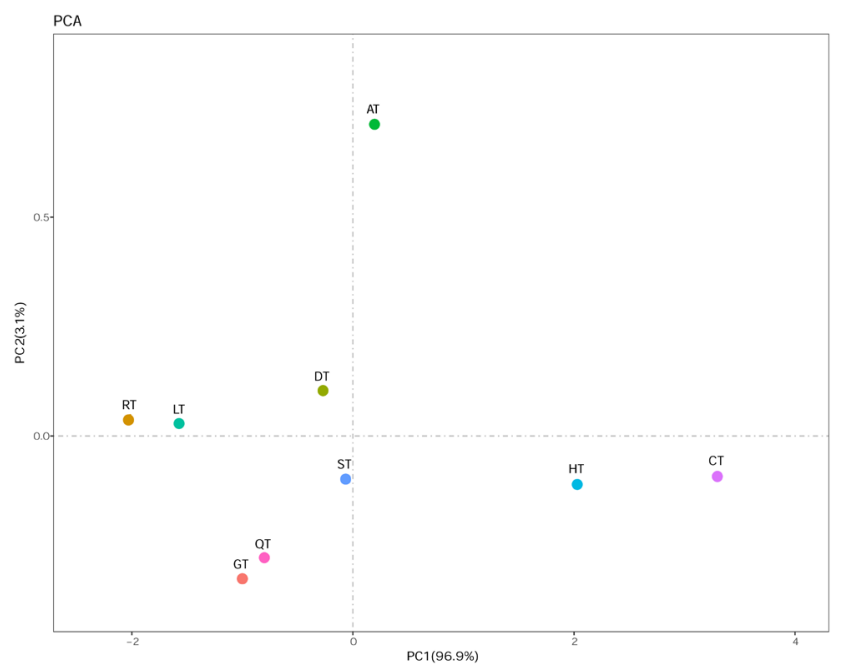

Figure 1. Principal component analysis (PCA). The 431-bp D-loop region of mtDNA was used to analyze the genetic diversity for all 1,201 sequences in Tibetan pigs (Diqing, $n=178$; Linzhi, $n=241$; Shannan, $n=91$; Changdu, $n=$ 90; Rkaze, $n=24 ;$ Aba, $n=70$; Ganzi, $n=133$; Hezuo, $n=268$; Qinghai, $n=$ 106). The cumulative contribution rate is in the parentheses.

type diversity (Hd), nucleotide diversity $(\mathrm{Pi})$ and average number of nucleotide differences $(\mathrm{K})$ are the basic parameters used to assess genetic diversity. PCA is a statistical procedure used to reduce the dimensionality of a dataset by transformation to a new set of variables (the principal components) to summarize the features of the data [10]. Genetic diversity was analyzed by PCA, and the results are shown in Figure 1. We extracted two principal components (PCA1 and PCA2) defined by principal component factor scores based on a components matrix from $\mathrm{Hd}, \mathrm{Pi}$, and $\mathrm{K}$. The cumulative contribution rate of PCA1 was $96.9 \%$, which indicates that it can reflect $96.9 \%$ of the original variables and can meet the application requirements. At this point, we made a decision to use PCA1 to reflect genetic diversity. We obtained the result from Figure 1, in that the highest genetic diversity was in Changdu Tibetan pigs (CT) followed by Hezuo Tibetan pigs (HT) and Aba Tibetan pigs (AT), while Rkaze Tibetan pigs (RT) have the lowest genetic diversity.

A total of 38 unique haplotypes were identified in nine Tibetan pig populations. The HT have the largest number of unique haplotypes (15) and the highest ratio of unique haplotypes (55.6\%). RT have no unique haplotypes (Figure 2). Thus, the HT population is the most distinctive, followed by the Ganzi Tibetan (GT) and Qinghai Tibetan pig (QT) populations.

\section{Genetic contribution analysis}

To synthesize genetic distinctiveness and diversity, Petit et al [11] proposed the approach of genetic contribution. The contributions of genetic diversity $\left(\mathrm{R}_{\mathrm{S}(\mathrm{k})}\right)$ and genetic distinctiveness $\left(\mathrm{R}_{\mathrm{D}(\mathrm{K})}\right)$ are combined to obtain the total genetic contribution

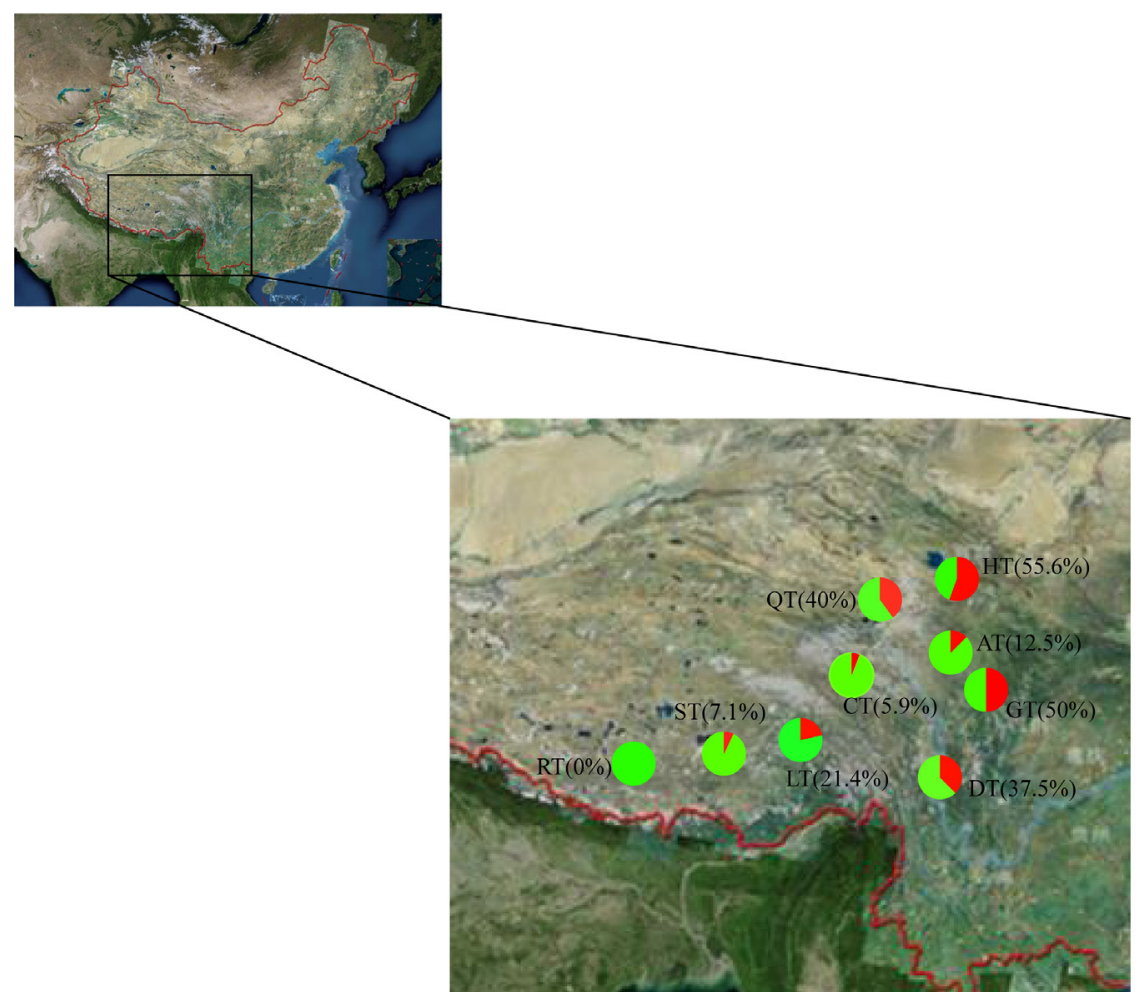

Figure 2. Comparison of the unique haplotype frequency of Tibetan pigs. Each pie chart shows the ratio of unique haplotypes and shared haplotypes among nine Tibetan pig populations. 
$\left(\mathrm{R}_{\mathrm{T}(\mathrm{K})}\right)$ of the kth population.

$$
\begin{aligned}
& \mathrm{R}_{\mathrm{S}(\mathrm{k})}=\frac{R_{k}}{n} \\
& \mathrm{R}_{\mathrm{D}(\mathrm{k})}=\sum_{i}^{R_{k}} \frac{n-n_{i}}{\mathrm{nn}_{i}} \\
& \mathrm{R}_{\mathrm{T}(\mathrm{k})}=\mathrm{R}_{\mathrm{S}(\mathrm{k})}+\mathrm{R}_{\mathrm{D}(\mathrm{k})}=\sum_{i}^{R_{k}} \frac{1}{n_{i}}
\end{aligned}
$$

where $\mathrm{n}$ represents the total number of populations studied, and $n_{i}$ represents the number of populations with the ith haplotype. Similarly, the rates of contribution attributed to genetic variation $\left(\mathrm{C}_{\mathrm{S}(\mathrm{K})}\right)$ and genetic distinctiveness $\left(\mathrm{C}_{\mathrm{D}(\mathrm{k})}\right)$ to the total genetic contribution rate $\left(\mathrm{C}_{\mathrm{T}(\mathrm{k})}\right)$ of the Kth population with $\mathrm{R}_{\mathrm{K}}$ haplotypes are obtained using the following formulas:

$$
\mathrm{C}_{\mathrm{RS}(\mathrm{k})}=\frac{R_{S(k)}-\grave{R}_{S}}{R_{T}}
$$

Table 2. Genetic contributions of nine Tibetan pig populations

\begin{tabular}{lcccc}
\hline Items & $\mathbf{h}$ & $\mathbf{R}_{\mathbf{S ( k )}}$ & $\mathbf{R}_{\mathbf{D}(\mathbf{k})}$ & $\mathbf{R}_{\mathbf{T}(k)}$ \\
\hline Aba Tibetan pig & 8 & 0.8889 & 1.4857143 & 2.3746032 \\
Ganzi Tibetan pig & 14 & 1.5556 & 7.9166667 & 9.4722222 \\
Diqing Tibetan pig & 16 & 1.7778 & 7.2301587 & 9.0079365 \\
Linzhi Tibetan pig & 14 & 1.5556 & 4.7261905 & 6.281746 \\
Shannan Tibetan pig & 14 & 1.5556 & 2.8190476 & 4.3746032 \\
Changdu Tibetan pig & 17 & 1.8889 & 3.4857143 & 5.3746032 \\
Rkaze Tibetan pig & 3 & 0.3333 & 0.031746 & 0.3650794 \\
Hezuo Tibetan pig & 27 & 3.0000 & 14.874603 & 17.874603 \\
Qinghai Tibetan pig & 10 & 1.1111 & 3.7634921 & 4.8746032 \\
\hline
\end{tabular}

$\mathrm{R}_{S_{(k)}, r}$ genetic diversity; $\mathrm{R}_{\mathrm{D}(\mathrm{k}),}$ genetic distinctiveness; $\mathrm{R}_{\mathrm{T}(\mathrm{k}),}$ total genetic contribution.

$$
\begin{aligned}
& \mathrm{C}_{\mathrm{RD}(\mathrm{k})}=\frac{R_{D(k)}-\bar{R}_{D}}{R_{T}} \\
& \mathrm{C}_{\mathrm{RT}(\mathrm{k})}=\frac{R_{T(k)}-\grave{R}_{T}}{R_{T}}
\end{aligned}
$$

where $\mathrm{R}_{\mathrm{T}}$ represents the total number of haplotypes, $\bar{R}_{S}=$ $\sum R_{S(k)} / n, \bar{R}_{D}=\sum R_{D(k)} / n, \bar{R}_{D}=\sum R_{T(k)} / n$. The total contribution rate $\mathrm{C}_{\mathrm{RT}(\mathrm{k})}$ can be partitioned into two components, $\mathrm{C}_{\mathrm{RS}(\mathrm{k})}$, which is the rate of contribution of the kth population due to its own diversity, and $\mathrm{C}_{\mathrm{RD}(\mathrm{k})}$, the contribution due to its divergence, i.e., $\mathrm{C}_{\mathrm{RT}(\mathrm{k})}=\mathrm{C}_{\mathrm{RS}(\mathrm{k})}+\mathrm{C}_{\mathrm{RD}(\mathrm{k})}, \sum C_{R S(k)}=0$, $\sum C_{R D(k)}=0, \sum C_{R T(k)}=0$.

The values obtained for $\mathrm{R}_{\mathrm{S}(\mathrm{k})}$ and $\mathrm{R}_{\mathrm{D}(\mathrm{K})}$ (and therefore $\mathrm{R}_{\mathrm{T}(\mathrm{K})}$ ) were highest for the HT and lowest for the RT (Table 2). The values of $\mathrm{C}_{\mathrm{S}(\mathrm{K})}, \mathrm{C}_{\mathrm{D}(\mathrm{K})}$, and $\mathrm{C}_{\mathrm{T}(\mathrm{k})}$ provide relative overall criteria for setting priorities for conservation of Tibetan pig populations. The highest values of $\mathrm{C}_{\mathrm{S}(\mathrm{K})}, \mathrm{C}_{\mathrm{D}(\mathrm{K})}$, and $\mathrm{C}_{\mathrm{T}(\mathrm{K})}$ were in $\mathrm{HT}$ (Figure 2) and lowest in RT.

\section{Shared haplotypes between Tibetan pigs and}

Table 3. Analysis of Tibetan pig haplotypes shared with indigenous pigs

\begin{tabular}{lccc}
\hline Population & Sc & S & Sc/S (\%) \\
\hline Aba & 68 & 70 & 97.14 \\
Ganzi & 112 & 133 & 84.21 \\
Diqing & 158 & 178 & 88.76 \\
Linzhi & 225 & 241 & 93.36 \\
Shannan & 87 & 91 & 95.60 \\
Changdu & 84 & 90 & 93.33 \\
Rikeze & 24 & 24 & 100.00 \\
Hezuo & 227 & 268 & 84.70 \\
Qinghai & 103 & 106 & 97.17 \\
\hline
\end{tabular}

Sc, number of Tibetan pigs sharing haplotypes with indigenous pigs; S, number of Tibetan pigs.

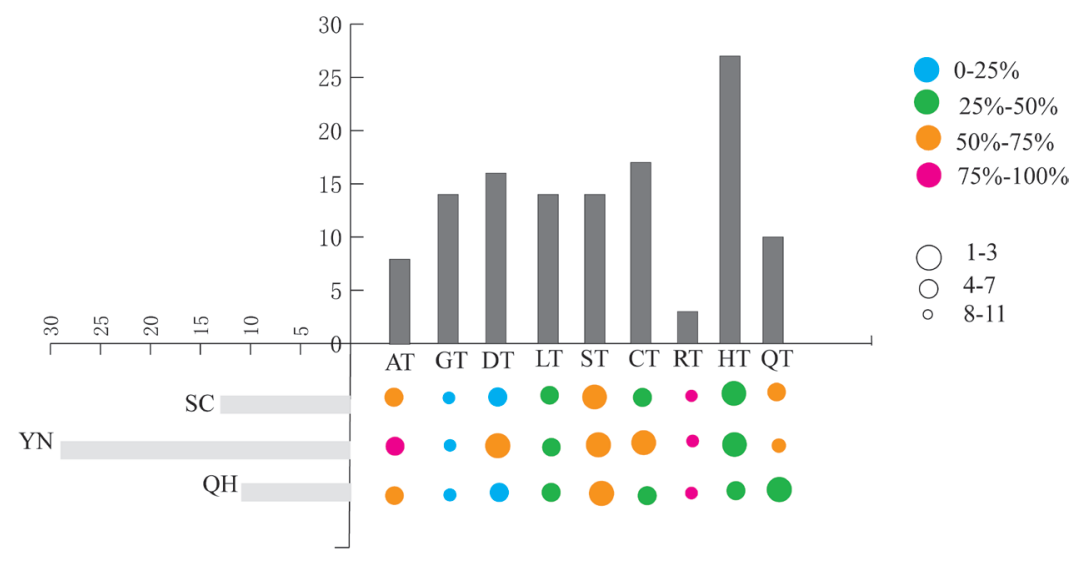

Figure 3. Shared haplotypes with indigenous pigs in Tibetan pigs. Each pie chart represents the shared haplotypes, and different sizes represent the number of shared haplotypes. Different colors represent the proportion of shared haplotypes in the total haplotypes of each Tibetan pig population. 


\section{indigenous breeds}

Haplotypes in 1201 individuals from nine Tibetan pig populations and 564 individuals from three indigenous breeds were identified (Table 1). Sixty haplotypes were identified in Tibetan pigs, and 40 haplotypes were identified in indigenous pigs. Twenty-three shared haplotypes were identified between indigenous and Tibetan pigs distributed among 511 indigenous and 1,088 Tibetan pigs. The shared haplotypes with number and degree of introgression to Tibetan pig haplotypes are shown in Figure 3. We can see that the GT haplotypes were minimally introgressed and the Diqing Tibetan (DT) haplotypes were minimally introgressed by the Sichuan indigenous pig and Qinghai indigenous pig haplotypes. The RT haplotypeswere greatly introgressed, and the AT haplotypes were greatly introgressed by the haplotypes of Yunnan indigenous pig. The percentage of the number of Tibetan pigs with shared haplotypes and the total of Tibetan pigs in each population $(\mathrm{Sc} / \mathrm{S})$ showed the degree of Tibetan pigs affected by indigenous pigs (Table 3). The average percentage of Sc/S was $92.7 \%$ and ranged from $84.7 \%$ to $100 \%$ (Table 3). Our data showed that Tibetan pigs were greatly impacted by indigenous breeds (from the Qinghai, Sichuan and Yunnan Provinces of China).

\section{DISCUSSION}

\section{Genetic diversity of the Tibetan pig population}

The genetic diversity of global livestock populations is declining [12]. Our study evaluated the genetic diversity in Tibetan pig populations. We analyzed the mtDNA D-loop for haplotypes of 1,201 Tibetan pig samples in nine populations (Table 1), and 60 haplotypes were identified, including 38 unique haplotypes. We also analyzed genetic diversity by PCA with a synthesized assessment score (Fz) score based on basic parameters $(\mathrm{Hd}, \mathrm{Pi}$, and $\mathrm{K})$. Hd reflects haplotype uniqueness in a population [13]. Pi measures the degree of polymorphism within a population [14]. Pi and $\mathrm{K}$ measure the degree of intrapopulation haplotype mutation [15]. The analysis showed that the genetic diversity in CT was highest and lowest in RT in nine Tibetan pig populations, which was consistent with the analysis of the parameters $\mathrm{Hd}, \mathrm{Pi}$, and $\mathrm{K}$. Fz was in the high range and higher on average, indicating that Tibetan pigs have a high status of genetic diversity in domestic pigs. In conclusion, the genetic diversity observed in Tibetan pigs highlights that it is an important genetic resource that is important for continued reproduction. Porcine genetic diversity could also be useful for sourcing future breeds for livestock production and supplementing biodiversity databasesaccumulated from populations and breeds around the world [16].

Genetic distinctiveness is an important factor that is used when populations are selected for conservation. The highest priority for conservation in the population often has the highest genetic distinctiveness [17]. A previous study selected distinct populations of Spring Monkey for priority conservation using evolutionarily significant units, which is a measure of genetic distinctiveness [18]. In this study, a total of 38 unique haplotypes were detected in nine Tibetan pig populations. The HT have the largest number of unique haplotypes (15) and the highest ratio of unique haplotypes (55.6\%). The RT have no unique haplotypes. The results suggest that the HT population has the highest priority for conservation in distinctive aspects, followed by the GT and QT populations.

\section{Genetic contribution}

Genetic diversity and genetic distinctiveness highlight two different aspects of genetic diversity; these two aspects are both important for the conservation of species. It is easy to misjudge using only one aspect, which is not conducive to the effective conservation of species. Therefore, considering the role of both aspects is necessary. Based on this, Petit et al [11] put forth the approach of genetic contribution, which considers genetic diversity and distinctiveness. This approach appears to be the most appropriate for selecting populations for conservation $[19,20]$. The contributions of genetic diversity $\left(\mathrm{R}_{S(\mathrm{~K})}\right)$ and genetic distinctiveness $\left(\mathrm{R}_{\mathrm{D}(\mathrm{K})}\right)$ are combined to obtain the total genetic contribution $\left(\mathrm{R}_{\mathrm{T}(\mathrm{K})}\right)$ of the kth population. $\mathrm{C}_{\mathrm{RS}(\mathrm{K})}$ and $\mathrm{C}_{\mathrm{RD}(\mathrm{k})}$ reflect the effects of population $\mathrm{k}$ in maintaining genetic diversity within the population and genetic divergence between populations, while $\mathrm{C}_{\mathrm{RT}(\mathrm{k})}$ reflects the combined effect of the two, which is the effect of maintaining the overall allelic richness of the species. The calculation of these three values reflects the measure of the relative contribution rate of the genetic diversity, distinctiveness and total genetic contribution by comparing the degree of difference between the genetic contribution rate of each population and the average genetic contribution among nine populations, based on

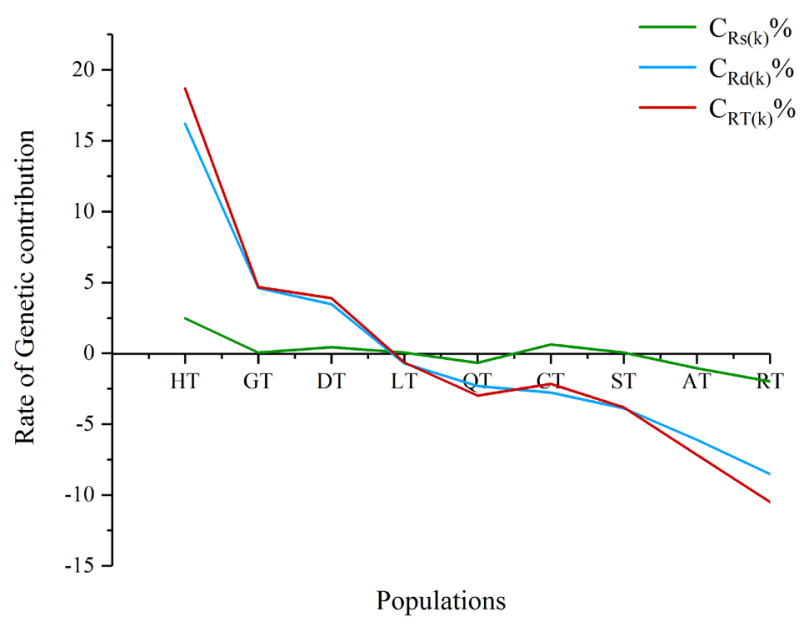

Figure 4. Rate of genetic contribution in nine Tibetan pig populations. 
genetic diversity, genetic divergence and total genetic contribution rate of population.

The order of priority conservation of a population based on genetic diversity and genetic distinctive were discussed respectively earlier in this study and did not take into account the combined effect of both aspects. Therefore, to fully consider the two factors of genetic diversity and genetic distinctiveness, the order of priority conservation of population was evaluated based on the model of genetic contribution rate.

As shown in Figure 4, the $\mathrm{C}_{\mathrm{RD}(\mathrm{k})}$ is generally consistent with the $\mathrm{C}_{\mathrm{RT}(\mathrm{k})}$ curve, while the $\mathrm{C}_{\mathrm{RS}(\mathrm{k})}$ is similar to the previous two curves, but the values are quite different. This indicates that the genetic distinctiveness has a greater contribution rate to the overall genetic effect, while the contribution rate of the genetic diversity effect is smaller; that is, the genetic distinctiveness has the greatest influence and the most sensitive effect on the overall genetic effect. Ranking of nine populations prioritized based on overall genetic effects, the overall genetic effect of HT, GT, and DT was positive, indicating that the genetic contribution of Tibetan pigs in these populations is higher than the average genetic contribution, and its presence increases the overall allele richness of Tibetan pigs and is worthy of conservation. The values in HT were higher than those of other populations, indicating that the HT population could contribute the most to improving the genetic variation and haplotype richness of Tibetan pigs, followed by the GT and DT populations.

In summary, based on the $\mathrm{C}_{\mathrm{RS}(\mathrm{k})}, \mathrm{C}_{\mathrm{RD}(\mathrm{k})}$, or $\mathrm{C}_{\mathrm{RT}(\mathrm{k})}$ values, the pre-existing population conservation value is large; in particular, those populations with positive $\mathrm{C}_{\mathrm{RS}(\mathrm{k})}, \mathrm{C}_{\mathrm{RS}(\mathrm{k})}$, or $\mathrm{C}_{\mathrm{RT}(\mathrm{k})}$ should be given priority protection.

\section{The introgression of Tibetan pigs}

Shared haplotypes were identified in $92.7 \%$ of Tibetan pigs on average, indicating that Tibetan pigs are severely introgressed by the indigenous pig breeds, which are distributed in the surrounding areas. In total, $38.3 \%$ (23/60) of haplotypes that were shared with indigenous pigs were identified in $90.6 \%(1,088 / 1,201)$ of Tibetan pigs. Shared haplotypes between indigenous and Tibetan pigs showed an unequal global distribution (Table 3). The frequency of shared haplotypes was lowest in HT, while it was highest in RT, where the Tibetan pig population was relatively greatly affected by indigenous pigs. To explore the conservation of Tibetan pig genetic resources from the perspective of introgression from indigenous breeds to Tibetan pigs, we should first consider the populations that are seriously affected by indigenous breeds. All individuals in the RT population analysis in this study shared haplotypes with indigenous breeds, indicating that the RT was more affected by indigenous influences.

\section{CONCLUSION}

Priority conservation order varies depending on the purpose of conservation. According to the principle of genetic diversity, the first priority in conservation is CT. According to the principle of genetic contribution rate, the first priority in conservation is HT. Tibetan pigs of different populations are threatened by indigenous pig breeds to varying degrees, RT is more affected by indigenous breeds, and the first priority in conservation is RT in introgression.

\section{CONFLICT OF INTEREST}

We certify that there is no conflict of interest with any financial organization regarding the material discussed in the manuscript.

\section{ACKNOWLEDGMENTS}

This work was supported by the Discipline construction fund project of Gansu Agricultural University (GAU-XKJS-2018048).

\section{REFERENCES}

1. Yang S, Zhang H, Mao H, et al. The local origin of the tibetan pig and additional insights into the origin of Asian pigs. PLoS One 2011;6:e28215. https://doi.org/10.1371/journal.pone. 0028215

2. Yang S-L, Wang Z-G, Liu B, et al. Genetic variation and relationships of eighteen Chinese indigenous pig breeds. Genet Sel Evol 2003;35:657. https://doi.org/10.1186/1297-9686-357-657

3. Larson G, Dobney K, Albarella U, et al. Worldwide phylogeography of wild boar reveals multiple centers of pig domestication. Science 2005;307:1618-21. https://doi.org/10.1126/ science. 1106927

4. Laval G, Iannuccelli N, Legault C, et al. Genetic diversity of eleven European pig breeds. Genet Sel Evol 2000;32:187. https:// doi.org/10.1186/1297-9686-32-2-187

5. Wu G-S, Yao Y-G, Qu K-X, et al. Population phylogenomic analysis of mitochondrial DNA in wild boars and domestic pigs revealed multiple domestication events in East Asia. Genome Biol 2007;8:R245. https://doi.org/10.1186/gb-20078-11-r245

6. Larson G, Liu R, Zhao X, et al. Patterns of East Asian pig domestication, migration, and turnover revealed by modern and ancient DNA. Proc Natl Acad Sci USA 2010;107:768691. https://doi.org/10.1073/pnas.0912264107

7. Zhang J, Yang B, Wen X, Sun G. Genetic variation and relationships in the mitochondrial DNA D-loop region of Qinghai indigenous and commercial pig breeds. Cell Mol Biol Lett 
2018;23:31. https://doi.org/10.1186/s11658-018-0097-x

8. Thompson JD, Gibson TJ, Plewniak F, Jeanmougin F, Higgins DG. The CLUSTAL_X windows interface: flexible strategies for multiple sequence alignment aided by quality analysis tools. Nucleic Acids Res 1997;25:4876-82. https://doi.org/10. 1093/nar/25.24.4876

9. Kumar S, Stecher G, Tamura K. MEGA7: Molecular evolutionary genetics analysis version 7.0 for bigger datasets. Mol Biol Evol 2016;33:1870-4. https://doi.org/10.1093/molbev/ msw054

10. Avise JC. Phylogeography the history and formation of species. Cambridge, MA, USA; London, England: Harvard University Press; 2000.

11. Petit RJ, Mousadik A, Pons O. Identifying populations for conservation on the basis of genetic markers. Conserv Biol 1998;12:844-55. https://doi.org/10.1111/j.1523-1739.1998. 96489.x

12. FAO. In: Rischkowsky B, Pilling D, editors. The State of the World's Animal Genetic Resources for Food and Agriculture. Rome, Italy: FAO; 2007. Available from: http://www.fao.org/ docrep/010/a1250e/a1250e00.htm

13. Zhang J, Jiao T, Zhao S. Genetic diversity in the mitochondrial DNA D-loop region of global swine (Sus scrofa) populations.
Biochem Biophys Res Commun 2016;473:814-20. https:// doi.org/10.1016/j.bbrc.2016.03.125

14. Nei M, Tajima F. DNA polymorphism detectable by restriction endonucleases. Genetics 1981;97:145-63.

15. Tajima F. Statistical method for testing the neutral mutation Hypothesis by DNA polymorphism. Genetics 1989;123:58595.

16. Li K, B Fan, S Zhao, et al. Analysis of diversity and genetic relationships between four Chinese indigenous pig breeds and one Australian commercial pig breed. Anim Genet 2000; 31:322-5. https://doi.org/10.1046/j.1365-2052.2000.00649.x

17. Moritz C. Defining "evolutionary significant units” for conservation. Trends Ecol Evol 1994;9:373-5.

18. Parker KM, Sheffer RJ, Hedrick PW. Molecular variation and evolutionarily significant units in the endangered gila topminnow. Conserv Biol 1999;13:108-116.

19. Chen XY, Lu HP, Sheng L, Li YY. Identifying populations for priority conservation of important species. Biodivers Sci 2002; 10:332-8.

20. Lu H, Shen L, Zhang X, Fan X, Chen X. Identifying populations for priority conservation II. Models based on haplotype richness and their applications in Ginkgo biloba. Acta Ecologica Sinica 2004;24:2312-6. 